Article

\title{
One-Stop Method to Measure Multiple Cardiac Performance Endpoints in Daphnia and Zebrafish by Kymograph
}

\author{
Kevin Adi Kurnia 1, , Ferry Saputra 1, ${ }^{1,}$, Marri Jmelou M. Roldan ${ }^{2}$, Agnes L. Castillo ${ }^{3}$, Jong-Chin \\ Huang ${ }^{4}$, Kelvin H.-C. Chen ${ }^{4, *}$, Hong-Thih Lai ${ }^{5, *}$ and Chung-Der Hsiao ${ }^{1,6,7, *}$ \\ 1 Department of Bioscience Technology, Chung Yuan Christian University, Chung-Li, 320314, Taiwan \\ 2 Faculty of Pharmacy and The Graduate School, University of Santo Tomas, Manila, 1008 Philippines \\ 3 Faculty of Pharmacy, The Graduate School and Research Center for the Natural and Applied Sciences, \\ University of Santo Tomas, Manila 1008 Philippines \\ 4 Department of Applied Chemistry, National Pingtung University, Pingtung 900391, Taiwan \\ 5 Department of Aquatic Biosciences, National Chiayi University, 300 University Rd., Chiayi 60004, Taiwan \\ 6 Center for Nanotechnology, Chung Yuan Christian University, Chung-Li 320314, Taiwan \\ 7 Department of Chemistry, Chung Yuan Christian University, Chung-Li, 320314, Taiwan \\ * equal contribution authors \\ * Correspondence: Kelvin H.-C. Chen (kelvin@mail.nptu.edu.tw); Hong-Thih Lai (htlai@mail.ncyu.edu.tw); \\ Chung-Der Hsiao (cdhsiao@cycu.edu.tw)
}

\begin{abstract}
Cardiovascular disease (CVD) is the number one cause of death worldwide. This condition resulted in huge research on CVD increasing the need for animal models suitable for the in vivo research. Daphnia and Zebrafish are good animal models for cardiovascular research due to their relative body transparency and easy culture property. Several methods have been developed to conduct cardiac performance measurement in Daphnia and Zebrafish. However, most of the methods only able to obtain heartbeat rate. The other important cardiac endpoints like stroke volume, ejection fraction, fraction shortening, cardiac output and heartbeat regularity must use other programs for measurement. To overcome this limitation, in this study, we successfully developed a one-stop ImageJ-based method using kymograph macros language that able to obtain multiple cardiac performance endpoints simultaneously for the first time. To validate its utility, we incubate Daphnia magna at different ambient temperatures and exposed zebrafish with astemizole to detect the corresponding cardiac performance alterations. In summary, the kymograph method reported in this study provides a new, easy to use, and inexpensive one-stop method on obtaining multiple cardiac performance endpoints with high accuracy and convenience.
\end{abstract}

Keywords: ImageJ; Kymograph; Cardiac Performance; Zebrafish; Daphnia

\section{Introduction}

Cardiovascular diseases (CVD) are the number one cause of death worldwide, accounting for 17.9 million or $31 \%$ of total annual worldwide deaths. This number is predicted to increase to 22.2 million annual deaths by 2030. Cardiovascular disease deaths are mainly caused by heart attacks and strokes, which are avoidable outcomes, mainly in developed countries. However, in low- and middleincome countries, more than three-quarters of deaths occurred due to these conditions [1]. As a result of these conditions, biomedical research on causes, prevention, and treatment of CVD remained active, producing hundreds of publications weekly. The sheer number of researches on CVDs increased the animal model needed for the research.

Zebrafish was proven to be a good vertebrate model for cardiovascular research because of several advantages it holds over the mammalian model. Physically, the human heart rate of 60-100 beats per minute (bpm) is closer to the zebrafish embryonic heart rate of 120-180 bpm, than the mouse heart rate of $300-600 \mathrm{bpm}$. Cardiovascular screening is mostly done in small zebrafish within the $1^{\text {st }}$ 
week of birth due to the transparent body of larval zebrafish, allowing non-invasive detection on the organ [2]. Zebrafish also produces a high offspring number, has a fast-embryonic development process, and short reproductive cycle. Due to its small size, zebrafish are easier to maintain and require little laboratory space. Furthermore, the zebrafish genome is fully sequenced and showed more than $70 \%$ similarity with human genes at protein level reflected in drug response, as the protein comprising drug binding targets is similar [3]. Due to this similarity, genes expressed in human disease are also expressed by zebrafish, providing a platform to model existing mutations in patients. These properties make zebrafish a fitting animal model for cardiovascular research [4]. In addition, zebrafish use for research also has fewer ethical restrictions compared to mammalian models [2].

Another animal fitting as an excellent cardiovascular research model is Daphnia magna. D. magna (water flea) is a well-known animal model because of its high sensitivity to environmental change and usually used as prescreening for toxicity assay [5]. Furthermore, a transparent body and large heart-to-body ratio is an advantage, allowing easier observation under a microscope [6]. D. magna has a myogenic heart, which is the intrinsic property of the mammalian heart and also known to have a similarity in cardiac response to components affecting heart rate in the mammalian animal model and even human [7-9]. All these advantages make D. magna became an excellent animal model to investigate the effect of some compounds on the cardiovascular system.

The current method for heartbeat measurement in Zebrafish and Daphnia involves record the dynamic pixel changes in the region of interest (ROI) by using either third-party or custom-designed software. Previously, we proposed a simple ImageJ-based method to measure cardiac rhythm in zebrafish embryos [10]. Heartbeat was recorded using a digital charged coupling device (CCD) mounted on a dissecting microscope, and the dynamic pixel changes were extracted from two consecutive frames. The low pixel intensity shows the presence of blood cells for heart relaxation, and high intensity shows the lack of blood cells for heart contraction. Jeong et al. also use a similar method to quantify the heart rate of D. magna by using ToupView 3.7 software [11,12]. Zhu et al. used a commercial Zebralab Blood Flow System (Viewpoint, France) [13]. While these methods proved to be useful to calculate heartbeat rate, those previous published methods suffer from the lack of accuracy if the transparency of the blood cell in the video is too high, and only few cardiac performance endpoints can be captured.

Kymograph is a 2-dimensional plot containing time and space information created from timelapse images of moving or pulsing objects of interest. It is able to quantify the movement velocity and trajectory of these objects. Kymograph is mainly used for axonal transport in nervous system research [14], microtubules dynamics, and particle movement [15]. This study aimed to develop a kymographbased method built in ImageJ platform [16] to provide a fast, easy-to-use and inexpensive one-stop method to obtain multiple cardiac performance endpoints with high accuracy for both Daphnia neonates and zebrafish embryos. To validate our method's accuracy, we compared the overall performance with a previously established method [10]. Furthermore, we also validate the result by recording at a different ambient temperature as D. magna used in this research have the ectothermic property $[17,18]$. We also uses astemizole to induce arrhythmia in zebrafish [10] and test whether our kymograph method is able to detect the corresponding cardiac performance changes.

\section{MATERIALS AND METHODS}

\subsection{Zebrafish and Daphnia maintenance and sample preparation}

In this study, wild type $A B$ strain zebrafish were used and maintained in a continuously filtered and aerated water system. According to previously reported protocol, the temperature was maintained at $26^{\circ} \mathrm{C}$ with a $10 / 14$ of dark/light cycle [19]. After harvested, the eggs were kept in an incubator and maintained at $28^{\circ} \mathrm{C}$ until 72 hours post-fertilization. Daphnia magna used in this study were cultured in an aquarium tank at $26^{\circ} \mathrm{C}$ dark/light cycle as the zebrafish. Daphnia used in this study has a body size of around $3-6 \mathrm{~mm}^{2}$. All protocols and procedure involving zebrafish and Daphnia were approved by the Committee for Animal Experimentation of the Chung Yuan Christian University (Approval No. 109001, issue date 15 January 2020). 


\subsection{Instrument setting for temperature control}

To test the effect of temperature in Daphnia, we used a temperature chamber (U 140A, BLAST, Japan) mounted on the inverted microscope (Sunny Optical Technology, Zhejiang, China). The temperature chamber was connected to the heater. Ice cube was used to lower the chamber's temperature while the heater will increase and maintain the temperature. The temperature was set to $15^{\circ} \mathrm{C}, 20^{\circ} \mathrm{C}, 25^{\circ} \mathrm{C}, 30^{\circ} \mathrm{C}$, and $35^{\circ} \mathrm{C}$ with one-minute acclimatization each time it reaches the desired temperature. The recording was done using a digital charge-coupled device (CCD) camera to capture the heartbeat of Daphnia.

\subsection{Astemizole treatment}

Astemizole stock solution was purchased from Enzo Life Science (Enzo Life Science, Inc., New York, USA). The stock solution was diluted to $40 \mu \mathrm{M}$ working solution at the time of recording. A mixture of $3 \%$ methylcellulose and $40 \mu \mathrm{M}$ astemizole in a 1:1 ratio was applied to zebrafish, resulting in a final astemizole concentration of $20 \mu \mathrm{M}$ while the control group was immobilized using $3 \%$ methylcellulose only. Heartbeat was recorded for 10 seconds after methylcellulose-astemizole treatment starting from minute 0 to minute 30 with 5 minutes interval for each recording. To record the zebrafish in astemizole treatment, we used the same camera setup with the temperature treatment with the exception of the temperature chamber.

\subsection{Video capturing and kymograph generation}

Initially, both zebrafish larvae and D. magna are positioned left-side facing upwards under the inverted microscope (Figure 2A and 2C) to facilitate for heartbeat recording. The videography was done using a high-speed CCD camera (Zgenebio, Taipei, Taiwan) capable of recording up to $200 \mathrm{fps}$ (frame per second) mounted to an inverted microscope (ICX41, Sunny Optical Technology, China). HiBestViewer (AZ Instrument, Taiwan) software was used to conduct high-speed video recording. Objective lens with 20x magnification was used to record Daphnia heart rate while 10x magnification was used for zebrafish larvae. The kymograph was generated from the video by using Dynamic Reslice tool on ImageJ Software [16]. Peak detection was done using Find Peaks Function on broadly applicable routines (BAR) plugin on ImageJ (Available online: https://imagej.net/BAR).

\subsection{Heart rate and heart rate variability measurement}

Heart rate (beat per minute) was measured by dividing 60 seconds with the average of time interval between peaks detected by BAR plugin. Heart rate variability was measured by using the Poincare plot plugin from OriginPro 2019 software (OriginLab Corporation, Northampton, MA, USA). The SD1 and SD2 of each individual were recorded and calculated statistically to determine the heart rate variability.

\subsection{Cardiac parameter endpoint calculation}

End diastolic diameter (EDD) 1 for short axis and for long axis and end systolic diameter (ESD) from both axis can be measured using the technique previously mentioned by Hsiao et al. [20]. The fractional shortening (FS) can be calculated using the following formula:

$$
F S=\frac{E D D 1-E S D 1}{E D D 1} \times 100 \%
$$

By assuming the ventricle is spheroid and the length of unknown diameter is the same as the shorter diameter, end diastolic volume (EDV) can be calculated using the following equation:

$$
\mathrm{EDV}=\frac{4}{3} \times \pi \times \frac{1}{2} E D D 2 \times \frac{1}{4} E D D 1^{2} \text { or } \frac{1}{6} \times \pi \times E D D 2 \times E D D 1^{2}
$$

End systolic volume (ESV) can also be calculated using the same equation. After obtained EDV and ESV, ejection fraction (EF) can be calculated using the equation: 


$$
E F=\frac{E D V-E S V}{E D V} \times 100 \%
$$

Stroke volume can also be calculated from known data by subtracting EDV with ESV.

$$
S V=E D V-E S V
$$

Stroke volume presents the volume of blood pumped each beat, therefore with the obtainable heart rate and stroke volume cardiac output can be calculated by the following formula:

$$
C O=S V \times \text { heart rate }
$$

\subsection{Statistics}

Data were compiled and analyzed statistically using GraphPad Prism 8 software (Graphpad Holdings, LCC, San Diego, USA). Significance level was set at $5 \%(p<0.05)$. Pearson correlation was used on normalized Daphnia heart rate data, while Wilcoxon test was used for Daphnia heart rate regularity as the data did not follow normal distribution assumption, the data was presented as mean \pm SEM. Effect of selected ROI positioning data on both atrium and ventricle (A-V) and ventricleatrium (V-A) interval measurement were calculated using kymograph and time series analyzer (TSA) method were calculated using Two-way ANOVA and presented as mean \pm SEM. Effect of astemizole treatment on zebrafish heartbeat were calculated using Two-way ANOVA. The data for multiple endpoints achievable using kymograph between control and astemizole-treated zebrafish were compared using unpaired t-test as the data did not follow normal distribution assumption.

\section{RESULTS}

\subsection{Overview of experimental workflow}

Although several cardiac rhythm measurement methods have been reported in zebrafish and Daphnia, most of them were only able to extract few cardiac physiology endpoints. In this study, we aimed to establish a one-stop, kymograph-based method to measure multiple cardiac physiology endpoints such as heart rate, ejection fraction, fractional shortening, stroke volume, cardiac output, and heartbeat variability. Two animal models of zebrafish and Daphnia magna were adopted since they are popular aquatic vertebrate and invertebrate models often used for cardiac physiology and toxicology studies. Initially, to obtain the unbiased data for fast heart beat rate nature, videography was performed by using a high-speed CCD mounted on an inverted microscope. The recording video (around $10 \mathrm{sec}$ ) was obtained in .mp4 format and needs to be converted to avi format using VirtualDub (Available online: virtualdub.org/download.html) software for downstream ImageJ processing. Kymographs were made from the region of interest (ROI), which are the heart on both species for heartbeat quantification. Due to anesthesia-free procedure used in this study, the specimen might move or struggle during recording, disrupting the quantification process. To overcome this limitation, we used ImageJ Stabilizer plugin [21] to stabilize image and reduce minormedium movement. The kymographs can be plotted, smoothened, and quantified using the Smoothed Plot Profile and Find Peaks tools in the BAR plugin in ImageJ [22]. Some macro languages were conducted in order to simplify the entire data collection process (see supplementary protocol). Finally, multiple cardiac physiology endpoints such as heart rate, ejection fraction, fractional shortening, stroke volume, and cardiac output can be obtained using mathematical calculation while heart rate variability can be analyzed using Poincare plot in OriginPro Software. The workflow of data processing for this research is schematically illustrated in Figure 1. 


\section{Image process}

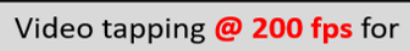

Video format conversion to avi format

Video stabilization using Image Stabilizer plugin (optional)

\section{Zebrafish}

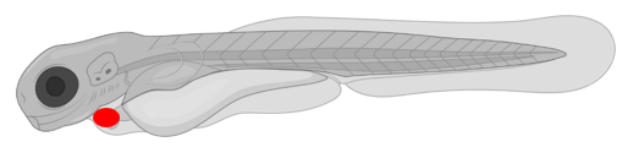

\section{Information extraction}

Use Kymograph plugin in ImageJ to analyze cardiac rhythm

Result plotting, smoothening, and interval detection in Image J using BAR Plugin

Data calculation using Microsoft Excel 2016

\section{Daphnia}

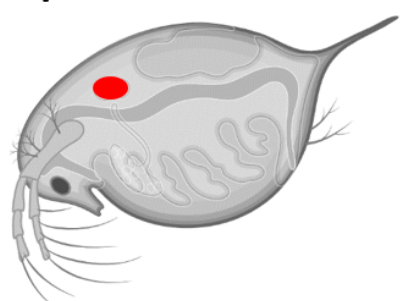

Obtainable Parameters

- Heart rate

- Heartbeat interval

- Fractional Shortening

- Ejection Fraction

- Stroke Volume

- Cardiac Output

- Heartbeat regularity

Figure 1. Overview of Experimental workflow for zebrafish and Daphnia heartbeat measurement by using kymograph method. Left panel showing image process pipeline, middle panel showing information extraction pipeline and right panel showing the cardiac parameters can be obtained by using this one-step kymograph method. The heart positions in zebrafish and Daphnia were highlighted by red dots in the bottom panel. .

\subsection{Measurement of heart rate by Kymograph in zebrafish and Daphnia}

Kymograph for zebrafish larvae and Daphnia heart chamber was made by drawing a line from the heart's inner part to the outermost area during diastole. The resulting kymograph can be adjusted using the Brightness/Contrast tool provided by ImageJ software to make a better visualization of heart movement during the beat for zebrafish larvae (Figure 2B) and Daphnia neonates (Figure 2D), respectively. The resulting kymograph shows the space and time relationship for heartbeat, and the time interval between two kymograph peaks can be defined as heartbeat interval. Quantification of zebrafish and Daphnia heartbeat starts by drawing a straight line from the leftmost side of the kymograph to the rightmost side (see supplementary protocol). The line must go through the ROI where heart movement or blood is present. Then heartbeat plot is made in ImageJ using the Plot Profile tool or by using the Smoothed Plot Profile tool included in the broadly applicable routine (BAR) plugin. Finally, a plot of time (x-axis) and gray value (y-axis) was made and the Find Peaks tool in the BAR plugin will automatically detect the timing of the peaks. 

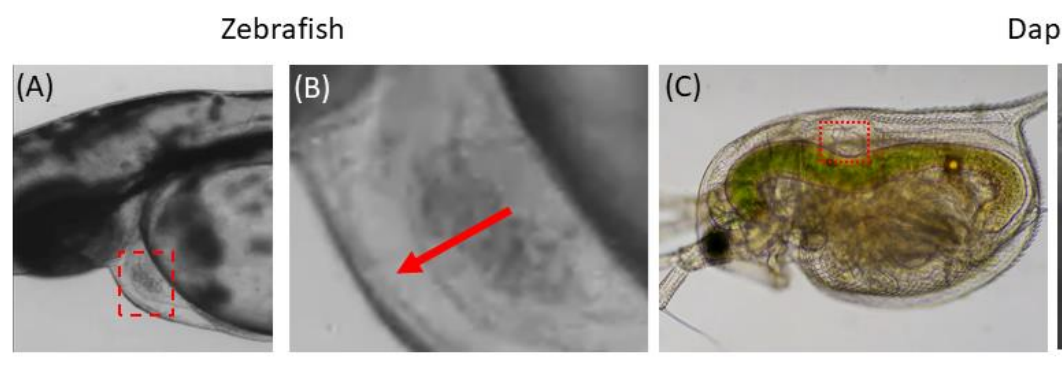

Daphnia
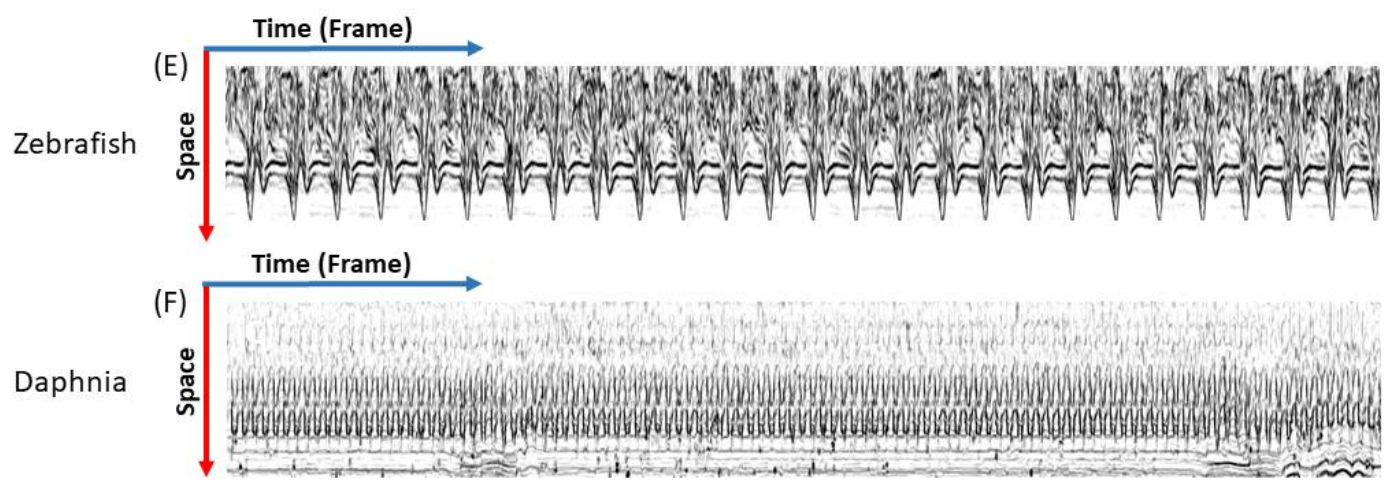

Figure 2. Principle for heartbeat measurement in zebrafish embryo by using kymograph. (A) The position used to calculate heartbeat in zebrafish embryo aged at 72 hours post-fertilization, (B) direction of the line made before dynamic resliced in zebrafish, $(C)$ the outlook of Daphnia showing the relative positions of heart, (D) direction of the line made before dynamic resliced in Daphnia, (E) the kymograph pattern of zebrafish heartbeat, and (F) the kymograph pattern of Daphnia heartbeat.

\subsection{Comparison of different measuring methods on heart rate variability in Daphnia magna}

Our previous reported Time Series Analyzer (TSA) method for cardiac rhythm measurement in zebrafish is based on the dynamic pixel change of blood within the heart chamber [10]. Once the selected ROI did not contain blood cell movement, this TSA-based ImageJ method might have some bias. On the other hand, the kymograph method reported here can measure either the blood cells within the heart chamber or heart chamber movement (contraction and relaxation), providing more flexible option choice for the users. To compare the utility and performance between TSA and kymograph methods, we raised Daphnia neonates in different temperature and their corresponding heartbeat rate and regularity were compared. Results show the heartbeat rate obtained from both methods display similar and consistent values at different testing temperature. This high consistency between TSA and kymography for heart rate measurement in Daphnia neonates were further justified through Pearson correlation statistical calculation (Figure 3A). This result shows kymograph capability to accurately detect high-speed heartbeat movement and distinguish the interval clearly within the plot.

Poincare plot is a good method to measure heart rate regularity by measuring the time interval between two consecutive beats in two dimensions. The standard deviation is defined as SD1 for the long axis and SD2 for the short axis. The heart rate regularity obtained from TSA and kymograph methods for Daphnia recorded in different temperature was compared. Interesting, both SD1 and SD2 showing higher variation in low temperature than high temperature groups for both analysis methods. Significant lower SD2 for kymograph was observed $(0.0116 \pm 0.0044$ of kymograph compared to $0.0155 \pm 0.0081$ of TSA), and significantly higher SD1 was observed $(0.0229 \pm 0.009$ of kymograph compared to $0.0312 \pm 0.0166$ of TSA). A consistent result is observed in SD1 and SD2 of Daphnia at all tested temperatures (Figure 3B). The most significant difference can be observed at ambient temperature of $25^{\circ} \mathrm{C}$, showing higher SD1 of $0.02383 \pm 0.007$ for kymograph v.s. $0.0069 \pm$ 0.0019 SD1 of TSA; and SD2 of $0.2083 \pm 0.0072$ v.s. $0.0046 \pm 0.0023$ SD2 for TSA. High variation shown 
by kymograph data demonstrated that kymograph method is more sensitive than the TSA method as it can detect the slightest change in the heart variability of Daphnia's heartbeat.

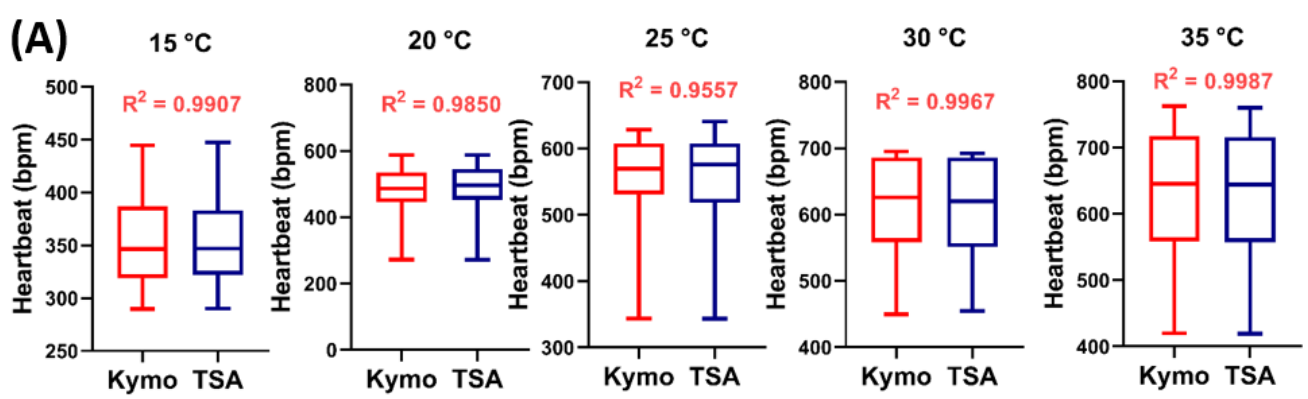

(B) Daphnia heart beat interval @ different temperature

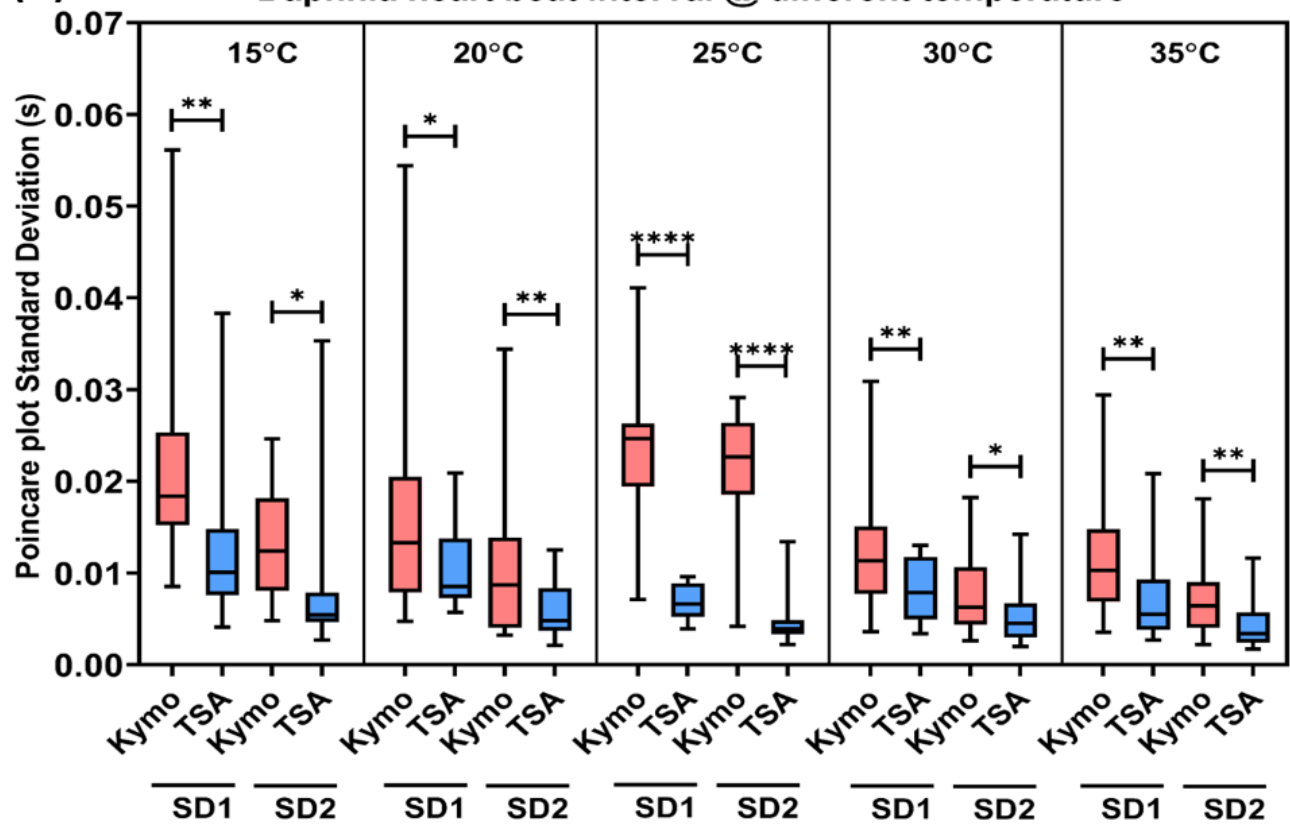

Figure 3. Comparison of kymograph (kymo) and time serial analyzer (TSA) methods on analyzing heart rate variability in Daphnia magna neonates cultured at different ambient temperatures. Two important indexes of SD1 and SD2 in the Poincare plot are used to measure heart rate regularity. Data were normalized for Pearson correlation calculation (A) and Wilcoxon test (B) presented as mean \pm $\operatorname{SEM}(\mathrm{n}=20) .\left(^{*} \mathrm{p}<0.5,{ }^{* *} \mathrm{p}<0.1,{ }^{* * * *} \mathrm{p}<0.0001, \mathrm{n}=20\right)$.

\subsection{Effects of region of interest (ROI) position on heartbeat regularity in zebrafish}

Based on the experience obtained from our previous TSA-based ImageJ method [10], we learned the selected ROI position play an essential role for cardiac rhythm detection. In this study, we would like to compare the performance between TSA and kymograph methods in different ROI positions. Zebrafish embryos were used for this validation, and three different ROI positions were tested as (1) middle position at both cardiac chambers, (2) low position for atrium and high position for ventricle and (3) high position for atrium and low position for ventricle (Figure 4A).

Since zebrafish has two cardiac chambers as atrium and ventricle, we measured the atriumventricle $(\mathrm{A}-\mathrm{V})$ and ventricle-atrium $(\mathrm{V}-\mathrm{A})$ intervals at three different positions based on either TSA or kymograph methods in zebrafish for cardiac rhythm comparison. The result shows that the different ROI positions indeed affect the analysis results. When using TSA method, significant differences were evident at low atrium and high ventricle measuring position (Low A-High $V$ ) for in A-V (Figure 4B) and or high atrium and low ventricle (High A-Low V) measuring position for V-A interval (Figure 4C). The kymograph-based method, on the contrary, showing more stable results with similar time intervals between all three testing positions (Figure 4B and $4 \mathrm{C}$ ). This result shows 
that the kymograph method is superior to the time series analyzer as it shows position-independent manner.

(A)

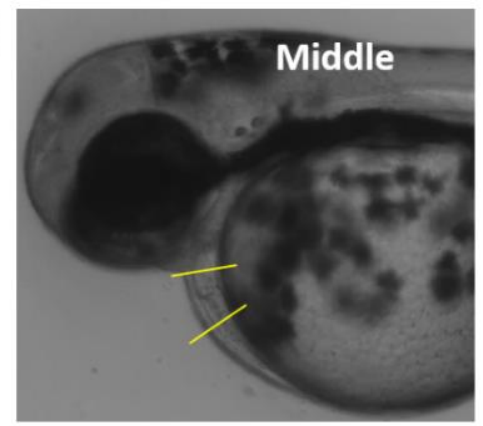

(B)

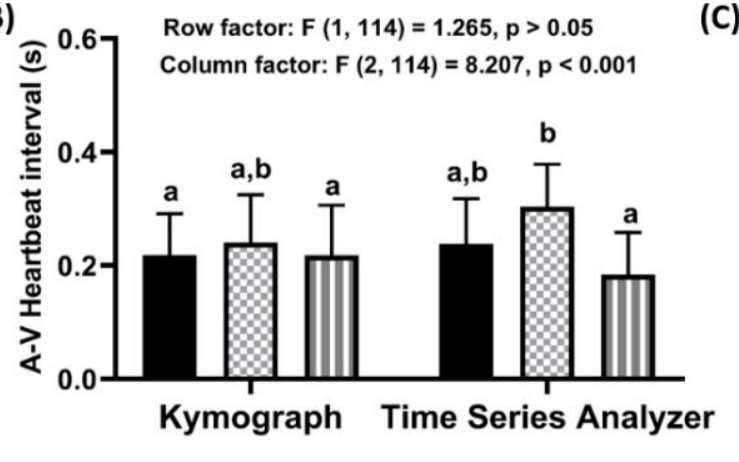

(C)
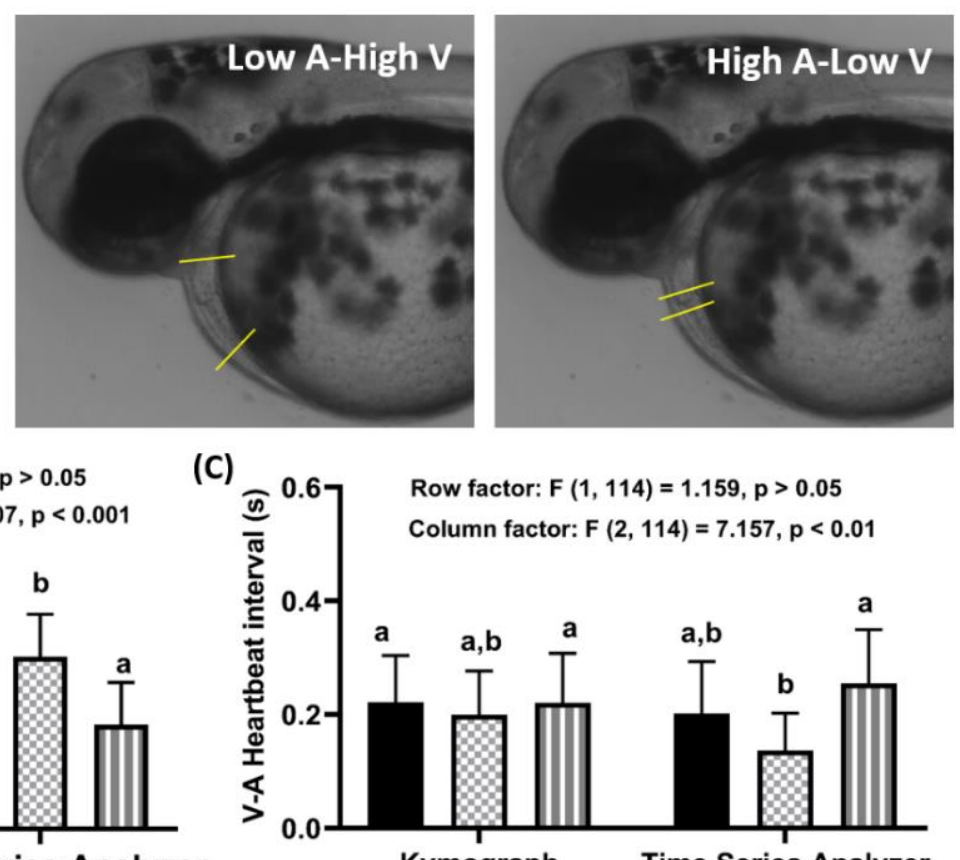

Kymograph
Time Series Analyzer

Middle

Low A-High V

High A-Low V

Figure 4. Effect of the selected ROI positioning on atrium-ventricle (A-V) and ventricle-atrium (V-A) interval measurement. (A) Three photos were showing the different positions of ROI when measuring heart rate in zebrafish embryos aged at 72 hours post-fertilization. There are middle ventricle-middle atrium, high ventricle-low atrium, and high atrium-low ventricle positions. Comparison of kymograph (kymo) and time series analyzer (TSA) methods to measure zebrafish (B) atrium-ventricle interval and $(C)$ ventricle-atrium interval. Data were calculated using a Two-way analysis of variance (ANOVA), presented as mean \pm SEM. $\left({ }^{*} \mathrm{p}<0.5, \mathrm{n}=20\right)$. Different letters $(\mathrm{a}, \mathrm{b})$ on the error bars represent the significant differences $(\mathrm{p}<0.05)$.

\subsection{Comparison of the heartbeat regularity after astemizole treatment in zebrafish}

Based on the optimized ROI position (middle position for both cardiac chambers), we would like to validate whether our developed kymograph method is able to evaluate potential cardiac arrhythmia. To archive this goal, astemizole (a second-generation antihistamines) was selected to induce arrhythmia in a time dependent manner according to our previous publication [10]. Compared to control group, astemizole treatment (at $20 \mu \mathrm{M})$ can induce a fast heart rate drop in both atrium and ventricle chambers from 10 min onwards until the end of the experiment (Figure 5A and $5 B)$. The lowest heart rate was observed in ventricle $(96 \pm 30 \mathrm{bpm})$ and in atrium $(104 \pm 25 \mathrm{bpm})$ at 20 minutes and $25 \mathrm{~min}$ after astemizole treatment, respectively (Figure 5A and 5B).

In addition to heart rate decline, astemizole treatment also induced heartbeat irregularity in zebrafish, which increases mostly in time dependent manner in both cardiac chamber (Figure 5C-5F). In the atrium, a significant difference was observed starting from 15 minutes after astemizole exposure (SD1 value of $0.0299 \pm 0.0226$ and SD2 value of $0.0219 \pm 0.0172$ ) compared to control group (SD1 value of $0.0096 \pm 0.0040$ and SD2 value of $0.0091 \pm 0.0041$ ). Both SD values kept showing significant difference until the end of the experiment on 30 minutes. However, in the ventricle, the result seems to vary between SD1 and SD2. SD1 shows a significant difference at 5, 20, and 25 minutes after astemizole treatment $(0.0487 \pm 0.0845)$ compared to control $(0.0161 \pm 0.0125)$.

For more detail evaluation of cardiac rhythm after astemizole treatment, we measured the A-V and V-A interval and found there are no significant change for A-V interval (Figure 5G) after 
astemizole treatment over time. However, a significant increment was observed on the V-A interval (Figure $5 \mathrm{H}$ ) from $0.166 \pm 0.075 \mathrm{~s}$ to $0.339 \pm 0.124 \mathrm{~s}$ at 15 minutes after astemizole treatment and 0.176 $\pm 0.069 \mathrm{~s}$ to $0.343 \pm 0.142 \mathrm{~s}$ at 20 minutes after treatment which give some insight that reduction of heart rate after incubation in astemizole mainly come from the increase of V-A interval. This result showing kymograph method can detect the change in cardiac rhythm after astemizole treatment in zebrafish. 


\section{(A) Heartbeat Ventricle}
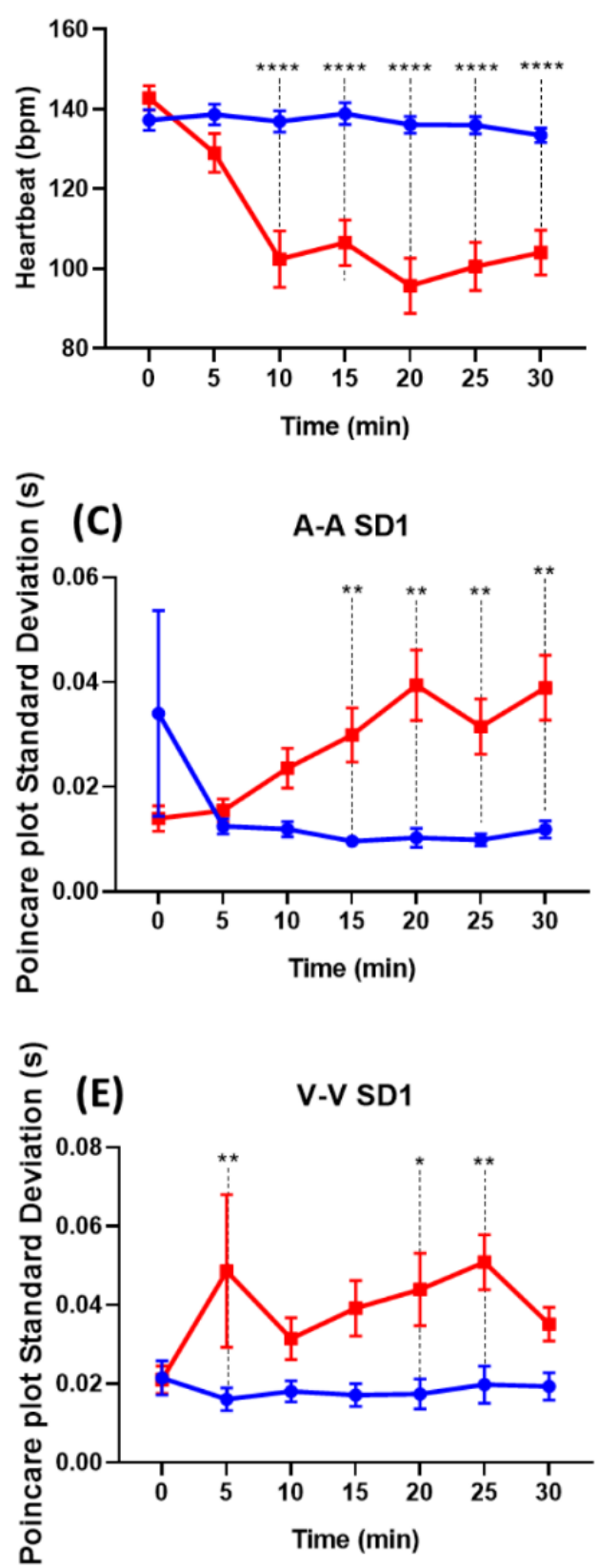

(G)

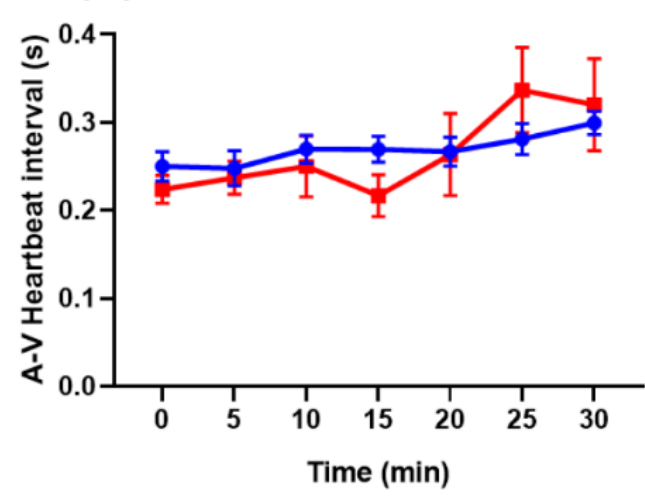

(B) Heartbeat Atrium
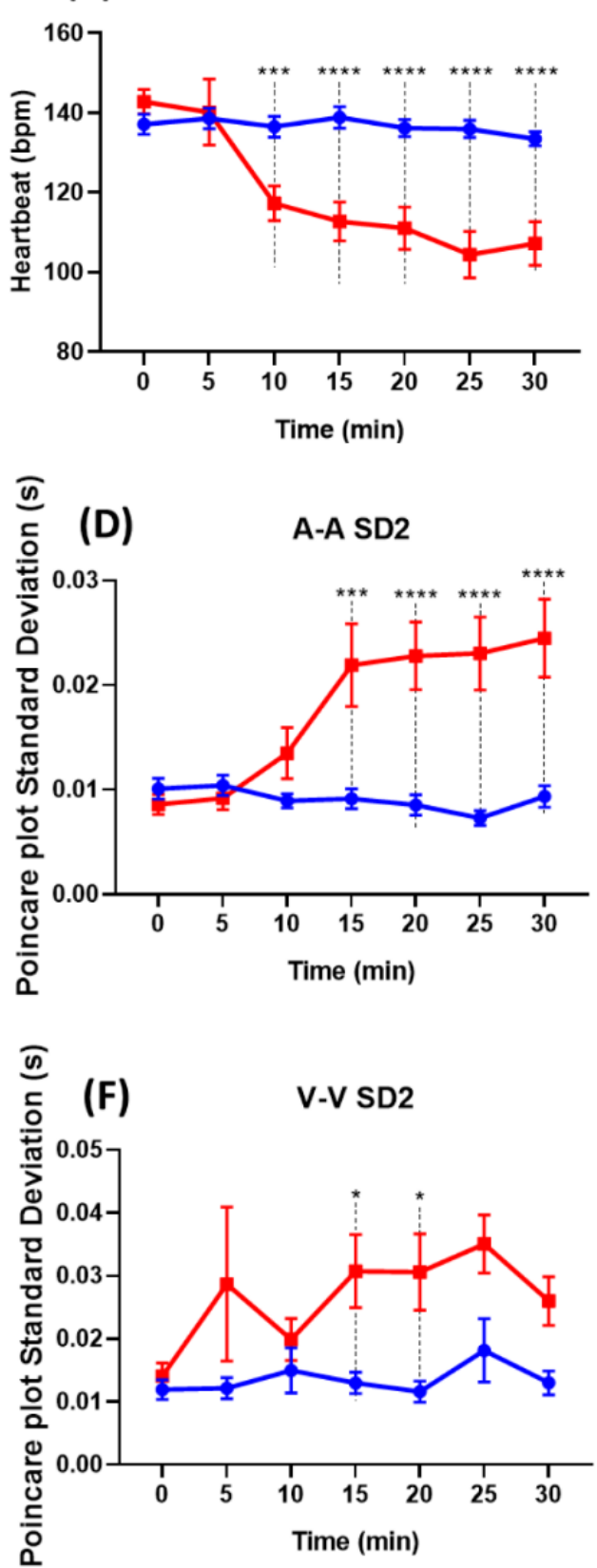

(H) V-A interval

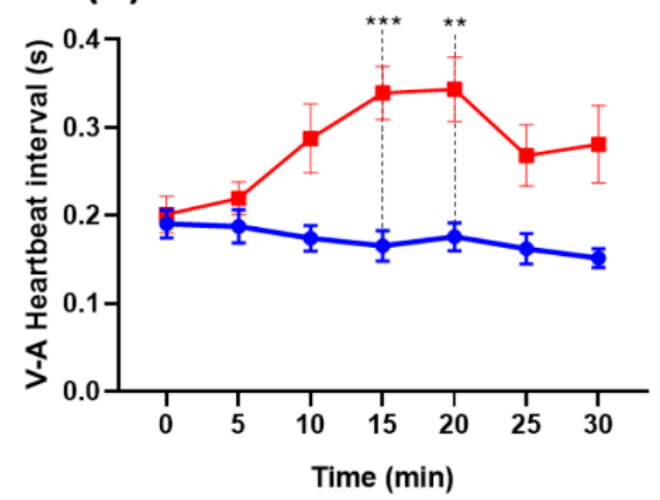


Figure 5. Effect of astemizole treatment on zebrafish cardiac parameters. (A) ventricle and (B) atrium heart rate, (C-F) atrium-atrium (A-A), and ventricle-ventricle (V-V) SD1 and SD2 calculated using a Poincare plot to present heart rate variability. (G-H) Atrium-ventricle (A-V) and ventricle-atrium (VA) interval of zebrafish heartbeat. Blue line $=$ control, red line=astemizole treatment. Zebrafish embryos were exposed to astemizole at 72 hours post-fertilization, and the corresponding heart rate changes were monitoring over time for 30 mins. Data were calculated using Two-way ANOVA and presented as mean \pm SEM. $\left({ }^{*} \mathrm{p}<0.5,{ }^{* *} \mathrm{p}<0.1,{ }^{* * *} \mathrm{p}<0.001,{ }^{* * * *} \mathrm{p}<0.0001, \mathrm{n}=20\right)$.

\subsection{Measurement of multiple cardiac performance endpoints by using Kymograph}

In addition to heart rate measurement, we next explored the possibility to use this kymographbased method to obtain other important heart parameters in a one-stop manner. These parameters include end-diastolic diameter (EDD), end-systolic diameter (ESD), heart period (HP), diastolic interval (DI) and systolic interval (SI) (Figure 6). For EDD and ESD measurement, a line can be drawn across the entire heart chamber at short and long axis (Figure 6A and 6B). EDD and ESD obtained from the heart short axis can be used for fractional shortening (FS) calculation. HP can be used to calculate the heart rate. Any significant changes within SI and DI can be considered as heartbeat irregularity. EDD and ESD from both short- and long-axes can be used to calculate stroke volume, fractional shortening, and ejection fraction. If combined with HP data, it can also calculate cardiac output (CO), as previously mentioned by Hsiao et al. [20].

To validate this tool's utility, we used parameters obtained from kymograph method to compare the cardiac physiology endpoints between control and astemizole-treated zebrafish embryos. Astemizole treatment increased the heart short-axis length and volume in both EDV and ESV while reducing heart rate, shortening fraction, ejection fraction, and cardiac output of zebrafish larvae. Furthermore, astemizole treatment also increases the ESV in zebrafish larvae. An increase in endsystolic volume means that the blood volume ejected from the heart chamber is low, showing the heart's lower contraction power (Table. 1). 


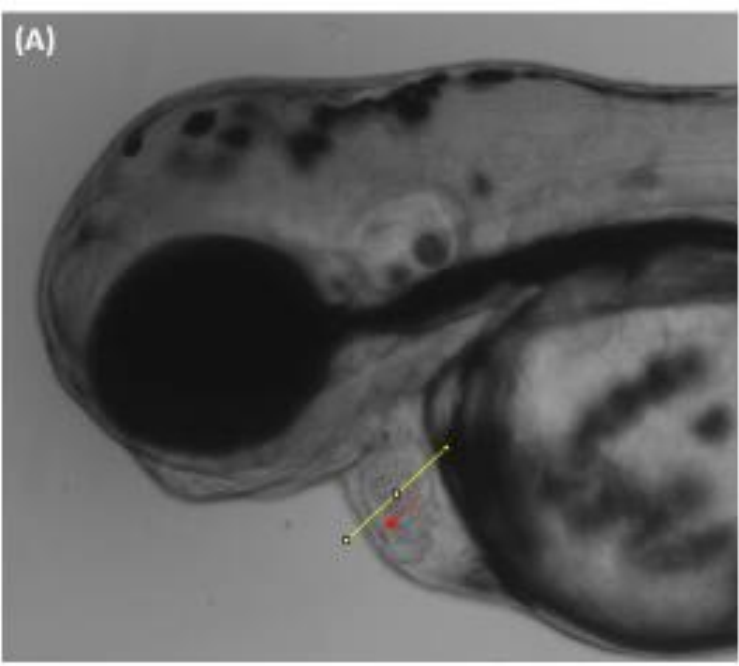

Ventricular short axis

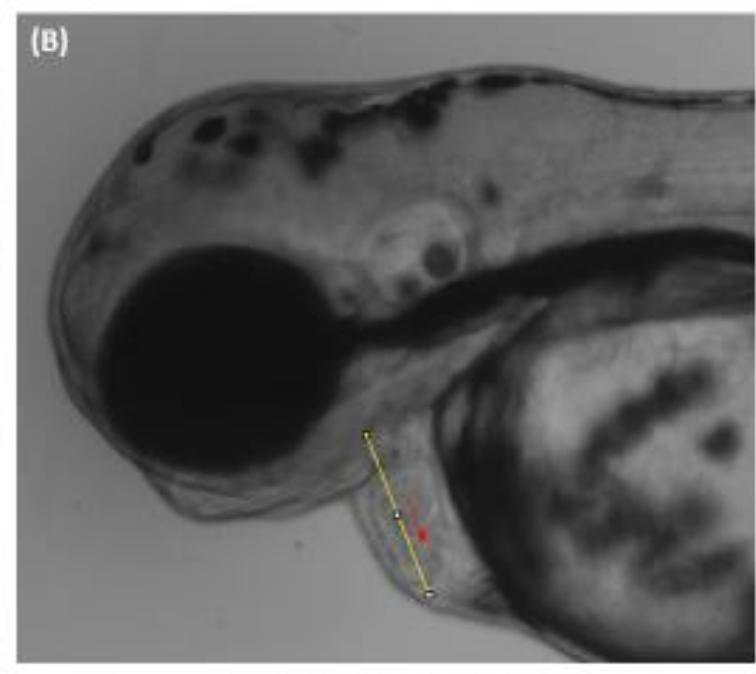

Ventricular long axis

Ventricular short axis Kymograph

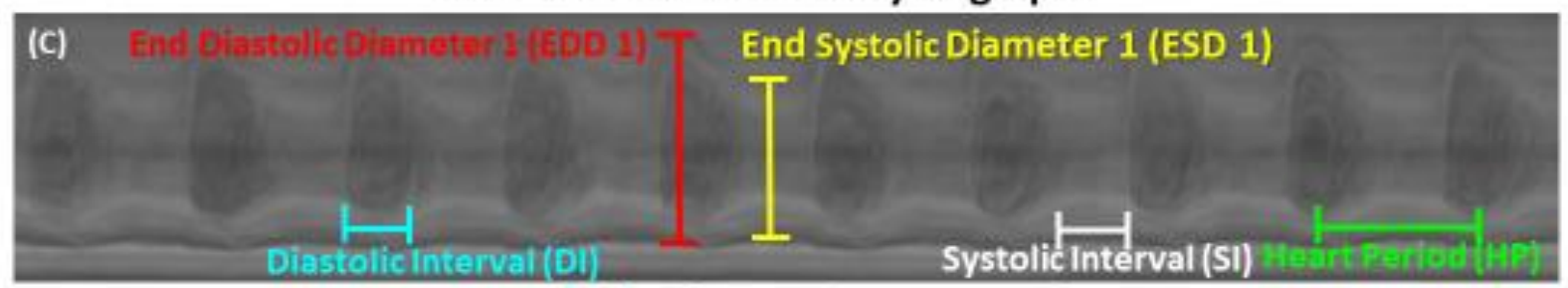

Ventricular long axis Kymograph

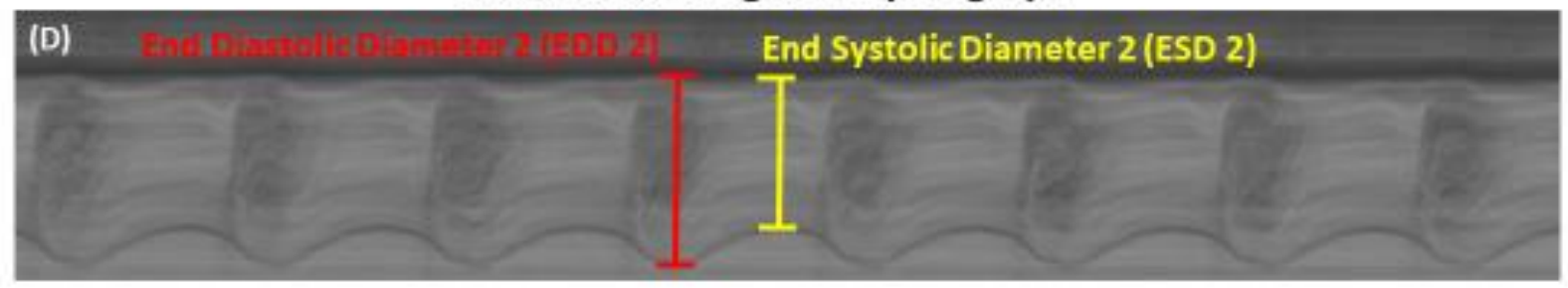

\section{(E) Normal}

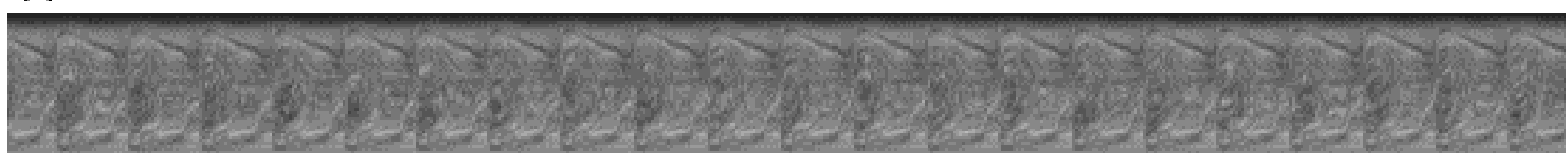

(F) Arrhythmic

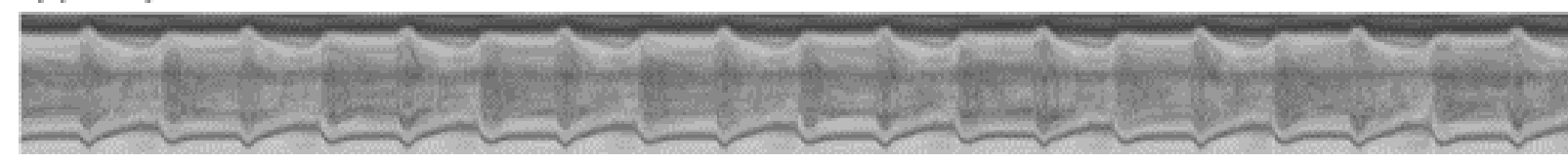

Figure 6. Cardiac physiology parameter endpoints in zebrafish aged at 72 hours post-fertilization measured by kymograph method. The position for kymograph measurement as (A) short-axis of the ventricle, (B) long-axis of the ventricle. The typical kymograph pattern obtained from the (C) shortaxis and (D) long-axis of ventricle. Some important parameters like end diastolic diameter (EDD, red color) and end systolic diameter (ESD, yellow color), heart period (HP, green color), diastolic interval (DI, blue color), and systolic interval (SI, white color) from either short axis or long axis were presented with sloid lines in different colors. The typical kymograph collected from ventricle (V) chambers of normal (E) and astemizole-induced arrhythmic (F) heart. 
Table 1. Comparison of multiple cardiac performance endpoints between control and astemizoletreated zebrafish aged at 72 hours post-fertilization.

\begin{tabular}{cccc}
\hline Parameters & Control & Astemizole $(\mathbf{2 0} \boldsymbol{\mu M})$ & $p$ \\
\hline End diastolic volume $\left(\mu \mathrm{m}^{3}\right)$ & $490,153.6 \pm 182,235.3$ & $773,517.1 \pm 324,727.3$ & $\leq 0.01$ \\
Long axis $(\mu \mathrm{m})$ & $140.81 \pm 20.18$ & $147.1 \pm 18.75$ & NS \\
Short axis $(\mu \mathrm{m})$ & $80.96 \pm 16.67$ & $98.42 \pm 17.85$ & $\leq 0.01$ \\
End systolic volume $\left(\mu \mathrm{m}^{3}\right)$ & $180,197.1 \pm 76,735.82$ & $524,531.6 \pm 338,855.3$ & $\leq 0.001$ \\
Long axis $(\mu \mathrm{m})$ & $106.77 \pm 17.16$ & $122.34 \pm 30.64$ & $\mathrm{NS}$ \\
Short axis $(\mu \mathrm{m})$ & $56.38 \pm 14.85$ & $85.31 \pm 22.18$ & $\leq 0.0001$ \\
Shortening fraction $(\%)$ & $30.72 \pm 13.87$ & $13.87 \pm 12.10$ & $\leq 0.0001$ \\
Ejection fraction $(\%)$ & $62.93 \pm 9.97$ & $35.70 \pm 22.65$ & $\leq 0.0001$ \\
Stroke volume $(\mathrm{pL} / \mathrm{beat})$ & $309.96 \pm 134.42$ & $248.99 \pm 151.00$ & $\mathrm{NS}$ \\
Heart rate $(\mathrm{bpm})$ & $133.42 \pm 7.84$ & $104.00 \pm 24.61$ & $\leq 0.0001$ \\
Cardiac output $(\mathrm{pL} / \mathrm{min})$ & $40,359.96 \pm 18,286.35$ & $25,351 \pm 16,238.36$ & $\leq 0.01$ \\
\hline
\end{tabular}

$p$ - value calculated statistically using unpaired $t$-test. $n=20$.

\section{DISCUSSION}

Although several methods have been established for heart rate analysis in aquatic animals, they are suffering from the drawbacks of limited usage, expensive instrument setting, or complicated operational procedures $[10,12,23]$ (summarized in Table 2). The most updated review on analyzing the pros and cons of current cardiac rhythm detection methods in zebrafish can be found in our recently review article [24]. Sampurna et al. [10] has developed a simple and cost-effective ImageJbased method to calculate cardiac rhythm in zebrafish. However, this stand-alone method is limited as it is only capable of calculating heartbeat rate, not other important cardiac parameters which manual measurement needed. This limitation promotes us to develop kymograph-based method to provide a more comprehensive way on data collection and processing. By utilizing several macro languages to facilitate image processing and semi-automated data calculation, we presented a more versatile kymograph-based method for cardiac performance evaluation in zebrafish larvae and Daphnia neonates for the first time.

Kymograph is a method to dissect the time and space relationship and been utilized to analyze cilia beating $[25,26]$ or vesicle transportation $[27,28]$. This method has been used to measure heartbeat in insect [29], crab [30] and freshwater mussel [31]. In Drosophila, Chakraborty et al. [32] used kymograph method for visualization of heart movement within a period, allowing them to obtain heart-related data such as EDD, ESD, HP, SI, and DI. The current kymograph-based method can be considered as a semi-automated tool for multiple heart parameter measurement by combining several tools like image stabilizer, BAR plugin and several macros to facilitate its overall performance. Similar approach has been reported by Collins et al. [33] to measure the cardiac arrhythmia in zebrafish pitx $2 c$ mutant by using MATLAB scripts. In this study, we provided an user-friendly ImageJ-based kymography tool and clearly demonstrated this new invented tool displays more versatile and higher accuracy than previously ImageJ-based method reported by Sampurna et al. [10] on measuring cardiac physiology.

Detection of heart chamber parameter using kymograph is based on the grey value on the time and space of the ROI. The presence of heart chamber wall or blood cell will increase the grey value on the time dimension. Thus, it is preferable to select ROI with higher contrast to the background as it can reduce the noise detected. By using our optimized instrument and software parameter settings presented in this study, we can detect the grey value over time of the heart wall with good contrast. In this study we use high speed CCD camera to record the heart chamber at $200 \mathrm{fps}$ and check the heart rate regularity. High frame rate is preferred in kymograph method as this method relies on the grey value caused by pixel color changes from frame to frame. High frame number will result in an increase of information and accuracy of the pixel changes. Higher frame rate is also more preferred during heart rate variability calculation as it will enable us to obtain the exact timing of the beat, especially with the animal with high heart rate like Daphnia. However, very high frame rate could 
result in more noise, resulting in a new problem, therefore depending on the heart rate, camera settings should be adjusted in order to record at suitable frame rate and obtain the optimal result [34].

Changes in heart rate can be caused by physiological and pathological factors, lifestyle factors, environmental factors, and neurophysiological factors [35]. Within these factors, temperature is a factor capable of inducing fast bradycardia or tachycardia [36]. Due to being ectothermic organism, changes in water temperature can significantly elevate Daphnia heart rate [37]. An increase in temperature will increase metabolic activity resulting in high cellular oxygen demand, which increases heart activity to fulfill the demand, while the opposite happens during low temperatures [38]. In this assay, we provide good evidences to show combination of high-speed videography and kymograph method can detect superfast heart beat in Daphnia when they are acclimated to high water temperature conditions (up to $600 \mathrm{bpm}$ ). In addition, more information regarding heart rate variability can be extracted by using kymograph method compared to TSA-based counterpart as kymograph method could sensitively detected more variability in heart rate compared to TSA method

In this study, we further validate the potential utility of our developed kymograph method that able to evaluate cardiac physiology alterations after waterborne astemizole exposure. Astemizole is a compound able to block outward repolarizing potassium channels to induce long QT syndrome. Overdosage of astemizole makes cytochrome-P450, a primary enzymatic system for detoxification, unable to metabolize the drugs result in prolonging the drug duration and causing adverse reactions. Long QT syndrome increases the possibility of an individual contracting a fatal ventricular arrhythmia called torsades de pointes (TDP) that might lead to sudden cardiac arrest [39]. In this study, we observed a faster decline of zebrafish heart rate starting from 10 minutes after astemizole treatment. However, a significant difference was observed starting from 15 minutes after treatment until the end of observation. According to high-speed videography, ventricular pumping was not observed 15 minutes after astemizole exposure; however, blood backflow from the ventricle to the atrium was observed. Furthermore, by using this one-step kymograph, we also observed significant changes in several cardiac parameters (like decline of EDV, SF, EF, HR and CO) after 30 minutes of astemizole treatment by blocking potassium channel in the heart muscle, disturbing heart muscle contractility and affecting the cardiac parameter and cardiac rhythm.

\section{CONCLUSION}

In conclusion, we provide a one-stop method for easy and inexpensive cardiac physiology assessment in two important aquatic models of zebrafish larvae and D. magna neonates. By using this kymograph-based ImageJ tool, we provided a method for scientists without experience in programming. The kymograph data is also comparable to the previous TSA- based method and competitive to other established methods. By using the image stabilizer, BAR plugin and developed macro, most of the works can be done semi-automatically in ImageJ platform. We believe this kymograph-based tool can provide research community an easy operated toolkit with multiple function for cardiac physiology and toxicology assessments. 
Table 2. Comparison of heartbeat measurement methods in Zebrafish larvae.

\begin{tabular}{|c|c|c|c|c|}
\hline $\begin{array}{l}\text { Published } \\
\text { methods }\end{array}$ & Main Region of Interest recording instrument & $\begin{array}{l}\text { Tracking } \\
\text { software }\end{array}$ & Animal model & Obtainable result \\
\hline Our study & $\begin{array}{c}\text { Inverted Microscope (ICX41, Sunny Optical } \\
\text { Technology, China) + High- Speed CCD } \\
\text { (Zgenebio, Taipei, Taiwan) } \\
\end{array}$ & ImageJ & $\begin{array}{l}\text { Zebrafish, AB Strain } \\
\text { and Daphnia magna }\end{array}$ & $\begin{array}{l}\text { Heart rate, fractional shortening, ejection fraction, } \\
\text { stroke volume, cardiac output, heartbeat interval, } \\
\text { and chamber-to-chamber interval }\end{array}$ \\
\hline $\begin{array}{l}\text { Sampurna et } \\
\text { al., } 2018[10]\end{array}$ & CCD mounted onto dissecting microscope & ImageJ & Zebrafish, AB Strain & Heart rate \\
\hline $\begin{array}{l}\text { Xing et al., } \\
2018 \text { [40] }\end{array}$ & $\begin{array}{c}\text { C3313/RHD2216 + C3100/RHD2000 (Intan } \\
\text { Technologies LLC) and Opticam + Stereo } \\
\text { Microscope }\end{array}$ & MATLAB & $\begin{array}{l}\text { Zebrafish, Strain not } \\
\text { mentioned }\end{array}$ & Heart rate \\
\hline $\begin{array}{l}\text { Lenning et al., } \\
2018 \text { [23] }\end{array}$ & $\begin{array}{l}\text { Electrocardiogram recording using MEA } \\
\text { membranes }\end{array}$ & LabVIEW & $\begin{array}{l}\text { Zebrafish, Strain not } \\
\text { mentioned }\end{array}$ & Heart rate, $\mathrm{P}$ waves, $\mathrm{QRS}$ complexes, and $\mathrm{T}$ waves \\
\hline $\begin{array}{l}\text { Jeong et al., } \\
2018 \text { [11] }\end{array}$ & Olympus-CKX41SF Microscope (Olympus) & Toupview 3.7 & Daphnia magna & Heart rate \\
\hline $\begin{array}{l}\text { Zhu et al., } 2018 \\
\text { [13] }\end{array}$ & Dissecting stereomicroscope (Olympus, Japan) & $\begin{array}{l}\text { Zebralab Blood } \\
\text { Flow System }\end{array}$ & Zebrafish, AB Strain & Heart rate \\
\hline $\begin{array}{l}\text { Ho et al., } 2007 \\
\qquad[41]\end{array}$ & $\begin{array}{c}\text { Axioplan microscope (Zeiss, Germany) with } \\
\text { Normaski optics and fluorescence, captured } \\
\text { using Sony digital camera }\end{array}$ & $\begin{array}{l}\text { Viewed frame by } \\
\text { frame (manual) }\end{array}$ & $\begin{array}{l}\text { Transgenic zebrafish } \\
\text { with GFP expression }\end{array}$ & Heart rate, stroke volume, cardiac output \\
\hline
\end{tabular}


Supplementary Materials: Video S1: Daphnia magna heartbeat at $25^{\circ} \mathrm{C}$ (7X slow motion). Video S2: Daphnia magna heartbeat at $35^{\circ} \mathrm{C}$ (7X slow motion). Video S3: Zebrafish heartbeat at 72 hours post-fertilization (7X slow motion). Video S4: Zebrafish heartbeat at 72 hours post-fertilization after exposed to astemizole (7X slow motion).

Author Contributions: Conceptualization, K.A.K. K.H.-C.C., H.-T.L. and C.-D.H.; methodology, K.A.K. and F.S.; software, F.S.; validation, M.J.M.R. and A.L.C.; formal analysis, J.-C.H.; investigation, K.A.K. and F.S.; resources, K.H.-C.C. and H.-T.L.; writing-original draft preparation, K.A.K., F.S., K.H.-C.C., H.-T.L. and C.-D.H.; visualization, C.-D.H.; supervision, and funding acquisition, K.H.-C.C., H.-T.L. and C.-D.H. All authors have read and agreed to the published version of the manuscript.

Funding: This research was funded by the Ministry of Science Technology, Taiwan, grant numbers MOST1072622-B-033-001-CC2 and MOST108-2622-B-033-001-CC2 to C.-D.H., and MOST107-2321-B415-001 to H.-T.L.

Conflicts of Interest: The authors declare no conflict of interest. The funders had no role in the design of the study; in the collection, analyses, or interpretation of data; in the writing of the manuscript, or in the decision to publish the results.

\section{REFERENCES}

1. WHO. Technical Package for Cardiovascular Disease Management in Primary Health Care. Hearts 2016, 76 .

2. Gut, P.; Reischauer, S.; Stainier, D.Y.R.; Arnaout, R. Little fish, big data: Zebrafish as a model for cardiovascular and metabolic disease. Physiological Reviews 2017, 97, 889-938, doi:10.1152/physrev.00038.2016.

3. Howe, K.; Clark, M.D.; Torroja, C.F.; Torrance, J.; Berthelot, C.; Muffato, M.; Collins, J.E.; Humphray, S.; McLaren, K.; Matthews, L. The zebrafish reference genome sequence and its relationship to the human genome. Nature 2013, 496, 498-503.

4. Bournele, D.; Beis, D. Zebrafish models of cardiovascular disease. Heart Failure Reviews 2016, 21, 803813, doi:10.1007/s10741-016-9579-y.

5. Guilhermino, L.; Diamantino, T.; Silva, M.C.; Soares, A. Acute toxicity test with Daphnia magna: an alternative to mammals in the prescreening of chemical toxicity? Ecotoxicology and environmental safety 2000, 46, 357-362.

6. Greene, M.; Pitts, W.; Dewprashad, B. Using Videography to Study the Effects of Stimulants on Daphnia magna. The American Biology Teacher 2017, 79, 35-40.

7. Campbell, A.K.; Wann, K.T.; Matthews, S.B. Lactose causes heart arrhythmia in the water flea Daphnia pulex. Comparative Biochemistry and Physiology - B Biochemistry and Molecular Biology 2004, 139, 225-234, doi:10.1016/j.cbpc.2004.07.004.

8. Villegas-Navarro, A.; Rosas-L, E.; Reyes, J.L. The heart of Daphnia magna: Effects of four cardioactive drugs. Comparative Biochemistry and Physiology - C Toxicology and Pharmacology 2003, 136, 127-134, doi:10.1016/S1532-0456(03)00172-8.

9. Gaikwad, N.; Panat, A.; Deshpande, M.; Ramya, K.; Khalid, P.; Augustine, P. Effect of shilajit on the heart of Daphnia: A preliminary study. Journal of Ayurveda and integrative medicine 2012, 3, 3.

10. Sampurna, B.P.; Audira, G.; Juniardi, S.; Lai, Y.-H.; Hsiao, C.-D. A simple imagej-based method to measure cardiac rhythm in zebrafish embryos. Inventions 2018, 3, 21.

11. Jeong, T.-Y.; Yoon, D.; Kim, S.; Kim, H.Y.; Kim, S.D. Mode of action characterization for adverse effect of propranolol in Daphnia magna based on behavior and physiology monitoring and metabolite profiling. Environmental Pollution 2018, 233, 99-108.

12. Jeong, T.-Y.; Kim, H.Y.; Kim, S.D. Multi-generational effects of propranolol on Daphnia magna at different environmental concentrations. Environmental Pollution 2015, 206, 188-194. 
13. Zhu, X.Y.; Wu, S.Q.; Guo, S.Y.; Yang, H.; Xia, B.; Li, P.; Li, C.Q. A Zebrafish heart failure model for assessing therapeutic agents. Zebrafish 2018, 15, 243-253, doi:10.1089/zeb.2017.1546.

14. Welzel, O.; Knörr, J.; Stroebel, A.M.; Kornhuber, J.; Groemer, T.W. A fast and robust method for automated analysis of axonal transport. European Biophysics Journal 2011, 40, 1061-1069, doi:10.1007/s00249-011-0722-3.

15. Smal, I.; Grigoriev, I.; Akhmanova, A.; Niessen, W.J.; Meijering, E. Microtubule dynamics analysis using kymographs and variable-rate particle filters. IEEE Transactions on Image Processing 2010, 19, 1861-1876, doi:10.1109/TIP.2010.2045031.

16. Rueden, C.T.; Schindelin, J.; Hiner, M.C.; DeZonia, B.E.; Walter, A.E.; Arena, E.T.; Eliceiri, K.W. ImageJ2: ImageJ for the next generation of scientific image data. BMC bioinformatics 2017, 18, 529.

17. Lee, L.; Genge, C.E.; Cua, M.; Sheng, X.; Rayani, K.; Beg, M.F.; Sarunic, M.V.; Tibbits, G.F. Functional assessment of cardiac responses of adult zebrafish (danio rerio) to acute and chronic temperature change using high-resolution echocardiography. PLoS ONE 2016, 11, 1-18, doi:10.1371/journal.pone.0145163.

18. Müller, M.F.; Colomer, J.; Serra, T. Temperature-driven response reversibility and short-term quasiacclimation of Daphnia magna. PLoS ONE 2018, 13, 1-14, doi:10.1371/journal.pone.0209705.

19. Avdesh, A.; Chen, M.; Martin-Iverson, M.T.; Mondal, A.; Ong, D.; Rainey-Smith, S.; Taddei, K.; Lardelli, M.; Groth, D.M.; Verdile, G. Regular care and maintenance of a zebrafish (Danio rerio) laboratory: an introduction. JoVE (Journal of Visualized Experiments) 2012, e4196.

20. Hsiao, C.-D.; Wu, H.-H.; Malhotra, N.; Liu, Y.-C.; Wu, Y.-H.; Lin, Y.-N.; Saputra, F.; Santoso, F.; Chen, K.H.-C. Expression and Purification of Recombinant GHK Tripeptides Are Able to Protect against Acute Cardiotoxicity from Exposure to Waterborne-Copper in Zebrafish. Biomolecules 2020, 10, 1202.

21. Li, K. The image stabilizer plugin for ImageJ. 2008.

22. Ferreira, T.; Hiner, M.; Rueden, C.; Miura, K.; Eglinger, J.; Chef, B. BAR Plugin for ImageJ. 2016.

23. Lenning, M.; Fortunato, J.; Le, T.; Clark, I.; Sherpa, A.; Yi, S.; Hofsteen, P.; Thamilarasu, G.; Yang, J.; Xu, $X$. Real-time monitoring and analysis of zebrafish electrocardiogram with anomaly detection. Sensors 2018, 18, 61 .

24. Santoso, F.; Farhan, A.; Castillo, A.L.; Malhotra, N.; Saputra, F.; Kurnia, K.A.; Chen, K.H.-C.; Huang, J.C.; Chen, J.-R.; Hsiao, C.-D. An Overview of Methods for Cardiac Rhythm Detection in Zebrafish. Biomedicines 2020, 8, 329.

25. Ott, C.; Lippincott-Schwartz, J. Visualization of live primary cilia dynamics using fluorescence microscopy. Current protocols in cell biology 2012, 57, 4.26. 21-24.26. 22.

26. Sanchez, T.; Welch, D.; Nicastro, D.; Dogic, Z. Cilia-like beating of active microtubule bundles. Science 2011, 333, 456-459.

27. O'Hagan, R.; Barr, M.M. Kymographic analysis of transport in an individual neuronal sensory cilium in Caenorhabditis elegans. In Cilia, Springer: 2016; pp. 107-122.

28. Mukherjee, A.; Jenkins, B.; Fang, C.; Radke, R.J.; Banker, G.; Roysam, B. Automated kymograph analysis for profiling axonal transport of secretory granules. Medical image analysis 2011, 15, 354-367.

29. Lagerspetz, K.; Perttunen, V. Effect of temperature on the periodic heart beat reversal and heart rate in Corethra plumicornis (Diptera). Journal of Insect Physiology 1962, 8, 621-625.

30. Florey, E. Studies on the nervous regulation of the heart beat in decapod Crustacea. The Journal of general physiology 1960, 43, 1061-1081.

31. Motley, H.L. Physiological studies concerning the regulation of heartbeat in freshwater mussels. 
Physiological Zoology 1934, 7, 62-84.

32. Chakraborty, M.; Llamusi, B.; Artero, R. Modeling of Myotonic Dystrophy Cardiac Phenotypes in Drosophila. Front Neurol 2018, 9, 473, doi:10.3389/fneur.2018.00473.

33. Collins, M.M.; Ahlberg, G.; Hansen, C.V.; Guenther, S.; Marin-Juez, R.; Sokol, A.M.; El-Sammak, H.; Piesker, J.; Hellsten, Y.; Olesen, M.S., et al. Early sarcomere and metabolic defects in a zebrafish pitx2c cardiac arrhythmia model. Proc Natl Acad Sci U S A 2019, 116, 24115-24121, doi:10.1073/pnas.1913905116.

34. Luo, J.; Lee, W.-N.; Wang, S.; Konofagou, E.E. P4A-2 an in-vivo study of frame rate optimization for myocardial elastography. In Proceedings of 2007 IEEE Ultrasonics Symposium Proceedings, New York, 31 October 2007; pp. 1933-1936.

35. Gubin, D.G.; Weinert, D.; Rybina, S.V.; Danilova, L.A.; Solovieva, S.V.; Durov, A.M.; Prokopiev, N.Y.; Ushakov, P.A. Activity, sleep and ambient light have a different impact on circadian blood pressure, heart rate and body temperature rhythms. Chronobiol Int 2017, 34, 632-649, doi:10.1080/07420528.2017.1288632.

36. Stauss, H.M. Heart rate variability. American journal of physiology. Regulatory, integrative and comparative physiology 2003, 285, R927-931, doi:10.1152/ajpregu.00452.2003.

37. Santoso, F.; Krylov, V.V.; Castillo, A.L.; Saputra, F.; Chen, H.-M.; Lai, H.-T.; Hsiao, C.-D. Cardiovascular Performance Measurement in Water Fleas by Utilizing High-Speed Videography and ImageJ Software and Its Application for Pesticide Toxicity Assessment. Animals 2020, 10, 1587.

38. Pirow, R.; Wollinger, F.; Paul, R.J. The sites of respiratory gas exchange in the planktonic crustacean daphnia magna: an in vivo study employing blood haemoglobin as an internal oxygen probe. Journal of Experimental Biology 1999, 202, 3089-3099.

39. Smith, S.J. Cardiovascular toxicity of antihistamines. Otolaryngology-Head Neck Surgery 1994, 111, 348354.

40. Xing, Q.; Huynh, V.; Parolari, T.G.; Maurer-Morelli, C.V.; Peixoto, N.; Wei, Q. Zebrafish larvae heartbeat detection from body deformation in low resolution and low frequency video. Medical $\mathcal{E}$ Biological Engineering E Computing 2018, 56, 2353-2365, doi:10.1007/s11517-018-1863-7.

41. Ho, Y.-L.; Lin, Y.-H.; Tsai, I.-J.; Hsieh, F.-J.; Tsai, H.-J. In vivo assessment of cardiac morphology and function in heart-specific green fluorescent zebrafish. Journal of the Formosan Medical Association 2007, $106,181-186$. 